\title{
Bacillus Calmette-Guerin infection following intravesical instillation: does the strain
}

matter?

\section{BCGites dans les suites d'instillations intravésicales : la souche de BCG importe-t-elle?}

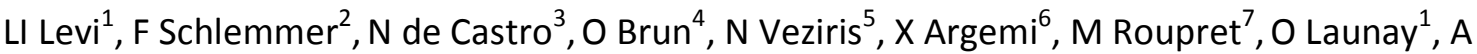 \\ Bergeron $^{8}$, and M. Groh ${ }^{1,9}$ for the BCG infection study group
}

1 Assistance Publique-Hôpitaux de Paris (AP-HP), Fédération d'infectiologie, Hôpital Cochin; Université Paris Descartes, Sorbonne Paris Santé, Paris, France.

${ }^{2}$ Unité de Pneumologie, Hôpitaux Universitaires Henri Mondor, DHU A-TVB, Assistance PubliqueHôpitaux de Paris (AP-HP), Université Paris-Est Créteil (UPEC), Créteil, France.

${ }^{3}$ Service de Maladies Infectieuses et Tropicales, Hôpital St Louis, Assistance Publique-Hôpitaux de Paris (AP-HP), Université Diderot, Paris, France.

${ }^{4}$ Service de Pneumologie, Clinique Medipôle Saint-Roch, Perpignan, France.

${ }^{5}$ Laboratoire de Bactériologie-Hygiène, Centre National de Référence des Mycobactéries et de la résistance aux antituberculeux, Hôpital de la Pitié-Salpêtrière, Assistance Publique-Hôpitaux de Paris (AP-HP), Sorbonne Université, UPMC Univ. Paris 06, CR7, Centre d'Immunologie et des Maladies Infectieuses, Team 13, INSERM U1135, Paris, France

${ }^{6}$ Service de Maladies Infectieuses et Tropicales, CHU de Strasbourg, Université de Strasbourg, France.

${ }^{7}$ Service d'Urologie, Hôpital de la Pitié-Salpêtrière, Assistance Publique-Hôpitaux de Paris (AP-HP), Sorbonne Université, Paris, France.

${ }^{8}$ Service de Pneumologie, Hôpital Saint-Louis, Assistance Publique-Hôpitaux de Paris (AP-HP), Université Diderot, Paris, France.

${ }^{9}$ Service de Médecine Interne, Hôpital Foch, Suresnes, France. 
BCG study group: Xavier Argemi, Anne Bergeron, François-Xavier Blanc, Philippe Blanche, Corinne Bouvier, Olivier Brun, Jean Patrice Calestrapout, Nathalie de Castro, Zoran De Jong, Sébastien Dufour, Aurélia Eden, Claude Erb, Philippe Fraisse, Jean-Charles Gagnard, Véronique Goudet, Cécile Goujard, Matthieu Groh, Aurélie Guigon, Laurent Hocqueloux, Tasnime Issoufaly, Akram Kazitani, Didier Laugros, Odile Launay, Vincent Le Moing, Nicolas Letang, Laura I. Levi, Frédéric Lucht, Laurence Mathé, Giovanna Melica, Amélie Ménard, Mathilde De Menthon, Laurence Moachon, Hervé Montsaint, Philippe Morlat, Jean-Marc Naccache, Didier Neau, Nathalie Nérault, Morgan Roupret, Frédéric Schlemmer, Thomas Sené, Grégory Verhoest, Nicolas Veziris, Dimitri Vordos, Fanny Vuotto, Benjamin Wyplosz, and Najdat Yaghi.

Corresponding author: Dr LI Levi, Fédération d'infectiologie, Hôpital Cochin; Université Paris Descartes, Sorbonne Paris Santé, Paris, France.

Email: levilaura@gmail.com

\section{Funding}

The authors declare no funding.

\section{Acknowledgements}

We are grateful to the Association Française d'Urologie (AFU), Société de Pathologie Infectieuse de Langue Française (SPILF), Société de Pneumologie de Langue Française (SPLF)/Groupe pour l'Enseignement et la Recherche en Pneumo-Infectiologie (GREPI), Amicale des Jeunes Internistes (AJI), and Centres de Lutte Antituberculeuse (CLAT) for helping with the case-by-case identification and data collection. 
Mots clés : BCG ; cancer de vessie

Keywords: BCG; urothelial carcinoma 
RÉSUMÉ

Objectifs. La BCG-thérapie est le traitement standard des tumeurs de vessie n'infiltrant pas la musculeuse. Aucune différence de tolérance entre les différentes souches de BCG n'a été rapportée.

Patients et méthodes. Une étude multidisciplinaire rétrospective française a été menée entre janvier 2013 et décembre 2016 pour identifier les cas de BCGites et comparer leur présentation clinique selon la souche utilisée.

Résultats. Quarante patients ont été inclus: (BCG RIVM 28; autres souches 8 ; inconnue 4). Les patients traités avec BCG-RIVM présentaient plus de miliaires pulmonaires $(71,4 \%$ vs $12,5 \%, p=0,005)$, moins de chocs septiques $(3,6 \%$ vs $50 \%$, $p=0,003$ ), des taux de transaminases plus bas (moyenne AST 65 vs $264 \mathrm{U} / \mathrm{I}, p=0,001$ ) et avaient moins recours à la réanimation $(7,1 \%$ vs $62,5 \%, p=0,003)$. L'évolution était superposable entre les deux groupes.

Conclusion. La souche de BCG pourrait influencer la fréquence et la sévérité d'une BCGite. 


\section{ABSTRACT}

Purpose. Intravesical BCG is the standard treatment of non-muscle-invasive bladder cancer. No difference has yet been reported in the safety profiles of the various BCG strains.

Methods. A nationwide multidisciplinary retrospective survey was conducted between January 2013 and December 2016 to identify cases of BCG infection and differentiate them based on the type of BCG strain used.

Results. Forty patients were identified (BCG RIVM 28; other strains 8; unknown 4). Patients treated with BCG RIVM were less severely ill, with fewer occurrences of septic shock ( $3.6 \%$ vs $50 \%, p=0.003)$ and ICU admission ( $7.1 \%$ vs $62.5 \%, p=0.003)$. A higher frequency of pulmonary miliaries $(71.4 \%$ vs $12.5 \%, p=0.005)$ but lower transaminase levels (mean AST 65 vs $264 \mathrm{U} / \mathrm{l}, p=0.001$ ) were observed in these patients. No difference in terms of recovery was reported.

Conclusion. The type of BCG strain could correlate with the frequency and severity of subsequent BCG infections. 


\section{INTRODUCTION}

Intravesical instillation of bacillus Calmette-Guerin (BCG) is the most effective adjuvant treatment of non-muscle invasive bladder cancer (NMIBC) after transurethral resection of the bladder (TURB) [1-7]. Side effects are usually mild and transient, although localized (1\%) or systemic ( $<1 \%$ to $4.7 \%)$ mycobacterial infections can occur [8-10]. These often lead to early treatment discontinuation, and thus negatively impact the disease course [11]. Among the eight available BCG-marketed strains, BCG Connaught, TICE, and Rijksinstituut voor Volksgezondheid en Milieuhygiene (RIVM) are the most commonly used. Data is conflicting regarding the strains' efficacy rates, and to date no difference has been identified in their safety profiles [12-17].

We performed a multicenter nationwide study to report on the clinical spectrum and therapeutic management of patients presenting with BCG infection following immunotherapy for NMIBC, in the context of a change of supplier (stockout of BCG Connaught at year end 2012, changed for BCG RIVM). We aimed to assess a potential link between the type of BCG strain and subsequent BCG infections.

\section{METHODS}

\section{Patients}

Between December 2013 and April 2016 three unrelated medical institutions noticed an unusually high number of BCG infections occurring over a short period of time in patients who received BCG intravesical instillation after TURB for NMIBC. Subsequently, thanks to collaborative networks (Association Française d'Urologie, 
Société de Pathologie Infectieuse de Langue Française, Société de Pneumologie de Langue Française, Amicale des Jeunes Internistes, Centres de Lutte Antituberculeuse), a nationwide retrospective survey was conducted to identify additional patients diagnosed with BCG infection over the same time period. This study complied with the Declaration of Helsinki and the French legislation regarding observational studies (Loi Huriet-Sérusclat 88-1138 and its subsequent amendments) in force at the time of the study.

\section{Definition of BCG infection}

BCG infections were defined as more than three days of high-grade fever and systemic and/or local manifestations (other than voiding symptoms) with no other plausible alternative diagnosis [9], occurring after BCG instillation for bladder cancer. Neither microbiological nor histological confirmation was needed to assess the diagnosis of BCG infection.

Assessments, outcome, and response to therapy

Collected data included demographic, clinical, biological, radiological, microbiological, and histological findings at the time of diagnosis and during followup. Recovery was defined as the resolution of all symptoms and biological abnormalities attributable to the BCG infection.

\section{Statistical analysis}

Descriptive statistics included the mean (SD) or median (range), as appropriate, for continuous variables, and frequency (percentage) for categorical variables. In each facility, the incidence rates were calculated using the ratio of the number of cases to the number of patients treated with BCG RIVM during the study period. Patient subsets were differentiated based on the type of BCG strain used. Univariate analysis 
included the chi-square or Fisher's exact test, as appropriate, to compare categorical variables and the non-parametric Mann-Whitney test to compare continuous variables. All tests were performed using GraphPad Prism version 7.0b 2016. A $p<0.05$ was considered significant.

\section{RESULTS}

\section{Patients}

When BCG Connaught stopped being marketed in France, 14 cases of RIVM-related BCG infections occurred in three unrelated hospitals (three in Cochin hospital, six in Saint-Louis hospital, five in Perpignan's Medipole clinic) with incidence rates of $7.3 \%$, $14.6 \%$, and $4.5 \%$ in these three facilities, respectively. Unlike the other two medical facilities, the BCG TICE strain was used concomitantly with the RIVM strain in the Saint-Louis hospital. No BCG infection was reported in the 19 patients (32\% of all patients) treated with this strain in that hospital over the same time period.

Twenty-six additional cases were identified thanks to the nationwide survey, among which 14 had been treated with BCG RIVM, eight with other strains (BCG Connaught, $n=5$; BCG TICE, $n=2 ; B C G$ SSI, $n=1$ ), and four with unknown strains. Hence, 28 patients were included in the BCG RIVM group and eight in the non-BCG RIVM group (Table 1).

\section{Baseline characteristics}

Median age at diagnosis was 69 years (IQR 14,3). Thirty-eight (95\%) patients were male and 25 (89.2\%) had high-grade NMIBC. General characteristics were identical in both groups, including the causes of underlying immunosuppression, the number of 
previous BCG instillations, and the traumatic nature of the last instillation (Table 1).

The clinical presentation at disease onset was highly heterogeneous with various TB presentations: miliary $(n=21)$, hepatitis $(n=8)$, tissue abscess $(n=3)$, isolated fever $(n=3)$, vascular involvement $(n=2)$, pelvic collection $(n=2)$, and adrenal necrosis $(n=1)$. Fever was constant, and 19 patients (47.5\%) had respiratory symptoms. Whole-body CT scans were performed on all patients: pulmonary miliary lesions were observed in 22 (55\%) patients, while other thoracic radiological features included pulmonary embolism $(n=3)$, mediastinal lymphadenopathy $(n=2)$, alveolar opacity $(n=4)$, and pulmonary nodule $(n=1)$. Patients treated with BCG RIVM were less severely ill, with fewer occurrences of organ failure $(10.7 \%$ vs $62.5 \%, p=0.006)$ and septic shock (3.6\% vs $50 \%, p=0.003)$, fewer ICU admissions ( $7.1 \%$ vs $62.5 \%$, $p=0.003)$ and a lower use of vasoactive drugs ( $3.6 \%$ vs $42.9 \%, p=0.018$ ). Patients treated with BCG RIVM more often presented with pulmonary miliary $(71.4 \%$ vs $12.5 \%, p=0.005$ ) but lower transaminase levels (mean AST 65 vs $264 \mathrm{U} / \mathrm{I}, p=0.001$ and ALT 76 vs $142 \mathrm{U} / \mathrm{I}, p=0.009$ ) than those treated with other strains.

\section{Microbiological and histological findings}

Overall, BCG infections were microbiologically confirmed in $11 / 40(27.5 \%)$ patients (Table 2 ) and were less frequent in the BCG RIVM group ( $17.9 \%$ vs $62.5 \%, p=0.024$ ). Respiratory samples were poorly sensitive. In patients presenting with cytopenia $(n=2)$ or $18^{F}-$ FDG PET/CT bone marrow involvement $(n=1)$, medullary culture was contributive. All patients with positive PCR on tissue biopsies $(2 / 5,40 \%)$ also had positive cultures on the same samples. Other PCR tests (blood, $n=1$; stools $n=1$ ) 
enabled the diagnosis for two patients who otherwise had no positive microbiological test.

Histological confirmation of the BCG infection was obtained in 12 (75\%) patients out of the 16 who underwent a biopsy. Overall, 14/18 biopsies performed led to the identification of epithelioid and gigantocellular granuloma. Besides bronchial biopsies (never contributive), all biopsy sites showed a good diagnostic sensitivity (ranging from $50 \%$ to $100 \%$ ) for the diagnosis of BCG infection (Table 2).

\section{Treatment and outcomes}

The management of patients was highly heterogeneous (Table 3) but comparable between groups. Overall, the combination of ethambutol (discontinued after two months), rifampicin, and isoniazid for six months was the most frequently prescribed regimen (26/40 patients, $65 \%)$.

Due to the severity of the initial clinical presentation $(n=16)$ or to general symptoms refractory to antimicrobial therapy $(n=5), 21$ patients $(52.5 \%)$ received an adjuvant treatment with prednisone (starting dose up to $1 \mathrm{mg} / \mathrm{kg} /$ day with gradual tapering) for a mean 2.6 months (range 0.25 - 8 months), among which six (29\%) experienced steroids-related adverse events.

Overall, there was no difference in treatment outcomes between strains (Table 3). Thirty-four patients (82.5\%) recovered from the BCG infection and two patients died (NMIBC progression and BCG-related respiratory failure despite steroid treatment, a single patient each).

\section{DISCUSSION}


BCG infection is a well-known complication after intravesical instillation for NMIBC. Yet, to our knowledge, the present study is the first to suggest that the type of BCG strain could correlate with the frequency and severity of subsequent BCG infections. Herein, rates of BCG infection were high in three facilities that used BCG RIVM and, overall, BCG RIVM-related infections were less severe than those occurring in patients treated with non-RIVM strains. Nevertheless, the overall mortality of this study is similar to that reported in the literature [9].

This work was initiated by several unrelated physicians who were struck by the high incidence of BCG infections occurring in their facility over a short period of time. Of note, all of the 14 initial cases occurred with the BCG RIVM strain despite the concomitant use of batches of BCG TICE in Saint-Louis Hospital, and no other case was reported after BCG RIVM stopped being used in Cochin hospital (September 2014).

We believe that host characteristics are not the main risk factor in our series as the BCG RIVM group was not massively immunosuppressed and because more than four weeks was observed between transurethral resection and BCG instillation in all cases. The four different batch numbers identified among patients with RIVMrelated BCG infection seem to rule out the hypothesis that a particular batch could be incriminated in these consecutive cases. As BCG RIVM comes with its own bladder catheter and as macroscopic hematuria was reported in $42.9 \%$ of cases reported in the present series (vs $1 \%$ to $34 \%$ in previous series [8-10]), local trauma might also be a plausible explanation. Of note, the decision of the BCG RIVM's supplier to change the instillation device in February 2016 gives credit, at least partly, to this assumption. 
Our study has a low sample size and limitations inherent to its retrospective design, namely missing data, loss to follow-up, and the lack of standardized diagnostic and therapeutic procedures. As there is no proper code for the diagnosis of BCG infection in the medical information system database, it is also likely that our study was not a comprehensive survey of all incident cases of BCG infection occurring in France over the study period.

Yet, this data arising from complementary collaborative networks supports the implementation of both basic and clinical studies to better decipher the interplay between BCG strains (and instillation devices) and patient outcomes. Hence, prospective studies are needed, especially as BCG Connaught production resumed at the end of 2016. As the batch number was reported in the medical files of only $15 / 40$ patients of the present series, our findings underline the opacity of our current drug traceability system, which should be considerably improved.

\section{CONTRIBUTION OF AUTHORS}

LIL, FS, and MG designed the protocol and wrote the first draft of the article; LIL, FS, NDC, OB, NV, XA, OL, MR, AB, and OL were involved in data collection and interpretation. LIL performed the statistical analyses. All authors were involved in critically revising the article and approved the final version to be submitted for publication.

\section{DECLARATION OF INTERESTS}

The authors declare no competing financial nor personal interests. 


\section{ETHICAL APPROVAL}

This study complies with the standard recommendations for clinical trials, the Declaration of Helsinki, and the French legislation regarding observational studies in force at the time of the study (Loi Huriet-Sérusclat 88-1138 and its subsequent amendments). 


\section{REFERENCES}

[1] van der Meijden AP, Steerenberg PA, de Jong WH, Debruyne FM. Intravesical Bacillus Calmette-Guérin treatment for superficial bladder cancer: results after 15 years of experience. Anticancer Res. 1991;11:1253-8.

[2] Denzinger S, Burger M, Walter B, Knuechel R, Roessler W, Wieland WF, et al. Clinically relevant reduction in risk of recurrence of superficial bladder cancer using 5-aminolevulinic acid-induced fluorescence diagnosis: 8-year results of prospective randomized study. Urology. 2007;69:675-9.

[3] Witjes JA, Redorta JP, Jacqmin D, Sofras F, Malmström PU, Riedl C, et al. Hexaminolevulinate-guided fluorescence cystoscopy in the diagnosis and follow-up of patients with non-muscle-invasive bladder cancer: review of the evidence and recommendations. Eur Urol. 2010;57:607-14.

[4] Chou R, Selph S, Buckley DI, Fu R, Griffin JC, Grusing S, et al. Intravesical Therapy for the Treatment of Non-Muscle-Invasive Bladder Cancer: A Systematic Review and Meta-analysis. J Urol. 2016.

[5] Babjuk M, Böhle A, Burger M, Capoun O, Cohen D, Compérat EM, et al. EAU Guidelines on Non-Muscle-invasive Urothelial Carcinoma of the Bladder: Update 2016. Eur Urol. 2016.

[6] Rouprêt $M$, Neuzillet $Y$, Masson-Lecomte $A$, Colin $P$, Compérat $E$, Dubosq $F$, et al. [CCAFU french national guidelines 2016-2018 on bladder cancer]. Prog Urol. 2016;27 Suppl 1:S67-S91.

[7] Morales A, Eidinger D, Bruce AW. Intracavitary Bacillus Calmette-Guerin in the treatment of superficial bladder tumors. J Urol. 1976;116:180-3. 
[8] Koya MP, Simon MA, Soloway MS. Complications of intravesical therapy for urothelial cancer of the bladder. J Urol. 2006;175:2004-10.

[9] Pérez-Jacoiste Asín MA, Fernández-Ruiz M, López-Medrano F, Lumbreras C, Tejido A, San Juan R, et al. Bacillus Calmette-Guérin (BCG) infection following intravesical BCG administration as adjunctive therapy for bladder cancer: incidence, risk factors, and outcome in a single-institution series and review of the literature. Medicine (Baltimore). 2014;93:236-54.

[10] González-Del Vecchio M, Ruíz-Serrano MJ, Gijón P, Sánchez-Somolinos M, de Egea V, García de Viedma D, et al. Differences between a probable and proven BCG infection following intravesical instillations: 16 years experience in a tertiary care hospital. Diagn Microbiol Infect Dis. 2016.

[11] Saint F, Irani J, Patard JJ, Salomon L, Hoznek A, Zammattio S, et al. Tolerability of bacille Calmette-Guérin maintenance therapy for superficial bladder cancer. Urology. $2001 ; 57: 883-8$

[12] Lamm DL, Stogdill VD, Stogdill BJ, Crispen RG. Complications of bacillus Calmette-Guerin immunotherapy in 1,278 patients with bladder cancer. J Urol. $1986 ; 135: 272-4$.

[13] Lamm DL, van der Meijden PM, Morales A, Brosman SA, Catalona WJ, Herr HW, et al. Incidence and treatment of complications of bacillus Calmette-Guerin intravesical therapy in superficial bladder cancer. J Urol. 1992;147:596-600.

[14] Lamm DL. Efficacy and safety of bacille Calmette-Guérin immunotherapy in superficial bladder cancer. Clin Infect Dis. 2000;31 Suppl 3:S86-90. 
[15] Steg A, Leleu C, Debré B, Boccon-Gibod L, Sicard D. Systemic bacillus CalmetteGuerin infection in patients treated by intravesical BCG therapy for superficial bladder cancer. Prog Clin Biol Res. 1989;310:325-34.

[16] Steg A, Adjiman S, Debré B. BCG therapy in superficial bladder tumours-complications and precautions. Eur Urol. 1992;21 Suppl 2:35-40.

[17] Witjes JA, vd Meijden AP, Witjes WP, Doesburg W, Schaafsma HE, Debruyne FM. A randomised prospective study comparing intravesical instillations of mitomycin-C, BCG-Tice, and BCG-RIVM in pTa-pT1 tumours and primary carcinoma in situ of the urinary bladder. Dutch South-East Cooperative Urological Group. Eur J Cancer. 1993;29A:1672-6. 
Table 1. Baseline characteristics of patients according to the type of BCG strain

Tableau 1. Caractéristiques initiales des patients selon la souche de BCG reçue

\begin{tabular}{|c|c|c|c|c|}
\hline & $\begin{array}{l}\text { Total } \\
\mathrm{n}=40\end{array}$ & $\begin{array}{c}\text { BCG-RIVM } \\
\mathrm{n}=\mathbf{2 8}\end{array}$ & $\begin{array}{l}\text { Non-BCG-RIVM } \\
n=8\end{array}$ & $p$ value \\
\hline \multicolumn{5}{|l|}{ General characteristics } \\
\hline Sex (Male), n (\%) & $38 / 40(95)$ & $26 / 28(92.9)$ & $8 / 8(100)$ & 0.99 \\
\hline Age (years), mean (SD) & $68 / 40(9.4)$ & $67 / 28(10.4)$ & $69 / 28(9.8)$ & 0.68 \\
\hline Immunosuppression, $\mathrm{n}(\%)$ & $12 / 40(30)^{*}$ & $8 / 28(28.6 \%)^{+}$ & $2 / 8(25)^{\dagger}$ & 0.99 \\
\hline High-grade NMIBC, n (\%) & $25 / 28(89.3)$ & $18 / 20(90)$ & $5 / 5(100)$ & 0.99 \\
\hline $\begin{array}{l}\text { Number of previous instillations (mean) / } \\
\text { data collected }\end{array}$ & $3-3 / 38$ & $2.5-3.3 / 26$ & $2.9-3.5 / 8$ & $0.12-0.82$ \\
\hline $\begin{array}{l}\text { Length between TUVR and BCG instillation (days), } \\
\text { median/data collected [IQR] }\end{array}$ & $77 / 22[43-83]$ & $\begin{array}{l}72.4 / 18[42- \\
73]\end{array}$ & $105 / 2[90-120]$ & 0.06 \\
\hline Recent urinary tract infection & $5 / 34(20.8)$ & $4 / 23(17.4)$ & $1 / 7(14.3)$ & 0.99 \\
\hline $\begin{array}{l}\text { Length between the last instillation and onset of } \\
\text { symptoms (days), median [IQR] }\end{array}$ & $1 / 39[0-5]$ & $1 / 28[0-4]$ & $2.5 / 8[0-285]$ & 0.58 \\
\hline$<1$ month, $\mathrm{n}(\%)$ & $35(87.5)$ & $25(89.3)$ & $6(75)$ & 0.22 \\
\hline$>3$ months, n (\%) & $4(10)$ & $2(7.1)$ & $2(25)$ & \\
\hline Traumatic instillation, n (\%) & $18 / 37(48.6)$ & $12 / 27(42.9)$ & $3 / 6(50)$ & 0.99 \\
\hline \multicolumn{5}{|l|}{ Symptoms and presentation, $\mathrm{n}(\%)$} \\
\hline Fever & $40 / 40(100)$ & $28 / 28(100)$ & $8 / 8(100)$ & 1 \\
\hline Organ failure & $8 / 40(20)$ & $3 / 28(10.7)$ & $5 / 8(62.5)$ & 0.006 \\
\hline Septic shock & $5 / 40(12.5)$ & $1 / 28(3.6)$ & $4 / 8(50)$ & 0.003 \\
\hline Dyspnea & $15 / 39(38.5)$ & $11 / 28(39.3)$ & $3 / 7(39.3)$ & 0.43 \\
\hline Cough & $11 / 39(28.2)$ & $9 / 27(33.3)$ & $2 / 8(25)$ & 0.99 \\
\hline
\end{tabular}




Expectoration
Oxygen therapy
Urinary tract symptoms
Cutaneous involvement
Splenomegaly
Hepatomegaly
Icterus
Laboratory findings, mean (SD)
C-reactive protein (mg/l)
AST (U/I)
ALT (U/I)
Alkaline phosphatase (U/I)
GGT (U/I)
Bilirubin ( $\mu$ mol/I)
Radiological findings, $\mathbf{n}$ (\%)
Miliary
Hepatic involvement
Pleural effusion
Prostatic involvement
Renal involvement
Pericardial effusion
Vascular involvement
Bone involvement

$\begin{array}{cccc}3 / 38(7.9) & 1 / 27(3.7) & 1 / 7(14.3) & 0.37 \\ 10 / 38(26.3) & 6 / 27(22.2) & 4 / 7(57.1) & 0.16 \\ 10 / 39(25.6) & 6 / 27(22.2) & 2 / 8(25) & 0.99 \\ 6 / 38(15.4) & 2 / 28(7.1) & 3 / 7(42.9) & 0.04 \\ 6 / 40(15) & 2 / 28(14.3) & 2 / 8(25) & 0.6 \\ 6 / 40(15) & 4 / 28(7.1) & 3 / 8(37.5) & 0.06 \\ 2 / 40(5) & 0 / 28(0) & 2 / 8(25) & 0.04 \\ & & & \\ 96.6 / 35(69) & 90 / 25(61) & 170 / 6(94) & 0.53 \\ 105.1 / 36 & 65.6 / 25(55) & 264.1 / 7(202) & 0.001 \\ (128) & & & \\ 97 / 36(94) & 75.9 / 25(63) & 203 / 7(128) & 0.009 \\ 243.1 / 32 & 196.1 / 23(147) & 443.5 / 6(419) & 0.19 \\ (245) & & & \\ 271 / 37(283) & 227.5 / 25(211) & 437.3 / 8(434) & 0.15 \\ 16.6 / 34(23) & 12.1 / 22(5) & 34.2 / 8(42) & 0.73 \\ & & & \\ 22 / 40(55) & 20 / 28(71.4) & 1 / 8(12.5) & 0.005 \\ 8 / 39(20.5) & 2 / 27(7.4) & 4 / 8(50) & 0.016 \\ 5 / 40(12.5) & 4 / 28(14.3) & 0 / 8(0) & 0.99 \\ 4 / 39(10.3) & 3 / 27(11.1) & 0 / 8(0) & 0.99 \\ 4 / 39(10.2) & 2 / 27(7.4) & 2 / 8(25) & 0.22 \\ 3 / 40(7.5) & 2 / 28(7.1) & 1 / 8(12.5) & 0.54 \\ 2 / 39(5.1) & 2 / 27(7.4) & 1 / 8(12.5) & 0.54 \\ 1 / 39(2.6) & 0 / 27(0) & 1 / 8(12.5) & 0.23\end{array}$




\section{Pathological findings}

Biopsy performed, $n$ (\%)

$16 / 39(41)$

75

$11(27.5)$
$8 / 27(29.6)$

50

5 (17.9)
$5 / 8(62.5)$

100

$5(62.5)$
0.12

0.11

0.02

* diabetes $(n=7)$, methotrexate and prednisone treatment for rheumatoid arthritis $(n=1)$, chronic kidney failure with glomerular filtration rate $<30 \mathrm{ml} / \mathrm{min}(\mathrm{n}=2)$, HIV infection $\left(C D 4>200 / \mathrm{mm}^{3}\right)$ ( $\left.n=1\right)$, myelodysplastic syndrome $(n=1)$

${ }^{+}$diabetes $(n=4)$, methotrexate and prednisone treatment for rheumatoid arthritis $(n=1)$, chronic kidney failure with glomerular filtration rate $<30 \mathrm{ml} / \mathrm{min}$ ( $\mathrm{n}=2$ ), HIV infection $\left(C D 4>200 / \mathrm{mm}^{3}\right)(\mathrm{n}=1)$

diabetes $(n=2)$

ALT: alanine aminotransferase; AST: aspartate aminotransferase; BCG: Calmette and Guérin bacillus; GGT: gamma-glutamyl transpeptidase; IQR: interquartile range; NMIBC: non-muscular invasive bladder cancer; RIVM: Rijksinstituut voor Volksgezondheid en Milieuhygiene; SD: standard deviation; TUVR: transurethral vesical resection. 
Table 2. Microbiological and pathological findings

Tableau 2. Données microbiologiques et anatomopathologiques

\begin{tabular}{|c|c|c|c|c|c|c|}
\hline & \multicolumn{2}{|c|}{ Total $n=40$} & \multicolumn{2}{|c|}{ BCG RIVM, n=28 } & \multicolumn{2}{|c|}{ Non-BCG RIVM, $n=8$} \\
\hline & $\begin{array}{c}\text { Perform } \\
\text { ed, } \\
\text { n (\%) }\end{array}$ & $\begin{array}{c}\text { Positive } \\
\text { sample, } \\
\text { n (\%) }\end{array}$ & $\begin{array}{c}\text { Perform } \\
\text { ed, } \\
\text { n (\%) }\end{array}$ & $\begin{array}{c}\text { Positive } \\
\text { sample, } \\
\text { n (\%) }\end{array}$ & $\begin{array}{c}\text { Performe } \\
\text { d, } \\
n(\%)\end{array}$ & $\begin{array}{c}\text { Positive } \\
\text { sample, } \\
\text { n (\%) }\end{array}$ \\
\hline \multicolumn{7}{|l|}{ Microbiological samples } \\
\hline Sputum smear & $20(50)$ & $2(10)$ & $16(57.1)$ & $1(6.3)$ & $3(37.5)$ & $1(12.5)$ \\
\hline Urinalysis & $23(57.5)$ & $5(21.7)$ & $13(46.4)$ & $2(15.4)$ & $6(75)$ & $1(12.5)$ \\
\hline Blood cultures & $21(52.5)$ & $2(9.5)$ & $14(50)$ & $1(7.1)$ & $5(62.5)$ & $1(12.5)$ \\
\hline Bronchoalveolar lavage & $12(30)$ & $0(0)$ & $10(35.7)$ & $0(0)$ & $1(12.5)$ & $0(0)$ \\
\hline Tissue biopsy and culture & $11(27.5)$ & $3(27.3)$ & $4(14.3)$ & $1(25)$ & $4(50)$ & $2(50)$ \\
\hline PCR on biopsy & $5(12.5)$ & $2(40)$ & $2(7.1)$ & $0(0)$ & $1(12.5)$ & $1(100)$ \\
\hline $\begin{array}{l}\text { Bone marrow aspiration } \\
\text { and culture }\end{array}$ & $3(7.5)$ & $3(100)$ & $2(7.1)$ & $2(100)$ & $1(12.5)$ & $1(100)$ \\
\hline Bronchoaspiration & $3(7.5)$ & $1(33.3)$ & $3(10.7)$ & $1(33.3)$ & $0(0)$ & $0(0)$ \\
\hline PCR on blood & $1(2.5)$ & $1(100)$ & $0(0)$ & $0(0)$ & $1(12.5)$ & $1(100)$ \\
\hline PCR on stool & $1(2.5)$ & $1(100)$ & $1(3.6)$ & $1(100)$ & $0(0)$ & $0(0)$ \\
\hline
\end{tabular}




\begin{tabular}{lcccccc} 
Liver & $6(15)$ & $5(83)$ & $2(7.1)$ & $1(50)$ & $3(37.5)$ & $3(100)$ \\
Abdominal mass & $2(5)$ & $2(100)$ & $0(0)$ & $0(0)$ & $0(0)$ & $0(0)$ \\
Lymph nodes & $2(5)$ & $2(100)$ & $1(3.6)$ & $1(100)$ & $1(12.5)$ & $1(100)$ \\
Cutaneous & $2(5)$ & $1(50)$ & $1(3.6)$ & $0(0)$ & $1(12.5)$ & $1(100)$ \\
Bronchial & $3(7.5)$ & $0(0)$ & $3(10.7)$ & $0(0)$ & $0(0)$ & $0(0)$ \\
Transbronchial & $1(2.5)$ & $1(100)$ & $1(3.6)$ & $1(100)$ & $0(0)$ & $0(0)$ \\
Bone marrow & $1(2.5)$ & $1(100)$ & $0(0)$ & $0(0)$ & $1(12.5)$ & $1(100)$ \\
Ovaries (adnexectomy) & $1(2.5)$ & $1(100)$ & $1(3.6)$ & $1(100)$ & $0(0)$ & $0(0)$ \\
& & & & & & \\
\hline
\end{tabular}

PCR: polymerase chain reaction 
Table 3. Patient management and outcome according to the type of BCG strain

Tableau 3. Prise en charge et devenir des patients selon la souche de BCG

\begin{tabular}{|c|c|c|c|c|}
\hline & $\begin{array}{l}\text { Total } \\
n=40\end{array}$ & $\begin{array}{c}\text { BCG-RIVM } \\
n=28\end{array}$ & $\begin{array}{c}\text { Non-BCG-RIVM } \\
n=8\end{array}$ & $p$ value \\
\hline \multicolumn{5}{|l|}{ Patient care } \\
\hline Length of hospitalization (days), median [IQR] & 23.6 [6.5-18.5] & 14 [7-17.5] & 25.5 [6.5-99] & 0.18 \\
\hline Need for intensive care, $\mathrm{n}(\%)$ & $7 / 40(17.5)$ & $2 / 28(7.1)$ & $5 / 8(62.5)$ & 0.003 \\
\hline Orotracheal intubation, n (\%) & $4 / 39(10.3)$ & $2 / 28(7.1)$ & $2 / 7(28.6)$ & 0.17 \\
\hline Use of vasoactive amines, $\mathrm{n}(\%)$ & $4 / 39(10.3)$ & $1 / 28(3.6)$ & $3 / 7(42.9)$ & 0.02 \\
\hline \multicolumn{5}{|l|}{ Treatment regimen } \\
\hline Antimycobacterial therapy & & & & 0.29 \\
\hline IR, n (\%) & $8 / 40(20)$ & 6/28 (21.4) & $0 / 8(0)$ & \\
\hline IRE, n (\%) & $25 / 40(62.5)$ & $17 / 28(60.7)$ & $7 / 8(87.5)$ & \\
\hline Other antibiotic combination, $\mathrm{n}(\%)$ & $7 / 40(17.5)$ & $5 / 28(17.8)$ & $1 / 8(12.5)$ & \\
\hline Duration of antimicrobial therapy (months), mean (SD) & $5.9(2.4)$ & $5.7(2)$ & $6.6(3)$ & 0.16 \\
\hline Use of glucocorticoids, n (\%) & $21 / 40(52.5)$ & $15 / 28(53.6)$ & $5 / 8(62.5)$ & 0.71 \\
\hline Duration of glucocorticoid therapy (months), mean (SD) & $2.6(2.2)$ & $2.6(2.4)$ & $3.1(1.9)$ & 0.62 \\
\hline Follow-up CT scan, n (\%) & $\mathrm{N}=23$ & $\mathrm{~N}=17$ & $\mathrm{~N}=4$ & 0.51 \\
\hline Stable or worse & $5 / 23(21.7)$ & $5 / 17(29.4)$ & $0 / 4(0)$ & \\
\hline Improved & $13 / 23(56.5)$ & 9/17 (52.9) & $2 / 4(50)$ & \\
\hline Normalized & $5 / 23(21.7)$ & $3 / 17(70.1)$ & $2 / 4(50)$ & \\
\hline \multicolumn{5}{|l|}{ Last follow-up } \\
\hline Time between BCG and last follow-up (days), median [IQR] & 478 [204-496] & 271 [188-476] & 5] $137[45-360]$ & 0.09 \\
\hline Recovery from BCG infection, $\mathrm{n}(\%)$ & $34 / 40(85)$ & $23 / 28(82.1)$ & $7 / 8(87.5)$ & 0.59 \\
\hline Death, $n(\%)$ & $2 / 40(5)$ & $1 / 28(3.6)$ & $1 / 8(12.5)$ & \\
\hline Resumption of urological treatments, $n$ (\%) & 17/34 (51.5) & 10/22 (45.4) & $5 / 8(62.5)$ & 0.42 \\
\hline Need for cystectomy, n (\%) & $6 / 30(20)$ & $4 / 22(18.2)$ & $2 / 5(40)$ & 0.30 \\
\hline Palliative care, $n$ (\%) & $5 / 31(16.1)$ & $2 / 21(9.1)$ & 1/7 (14.3) & 0.99 \\
\hline Remission of malignancy, $n$ (\%) & $17 / 30(58.6)$ & $13 / 21(61.9)$ & $3 / 5(60)$ & 0.99 \\
\hline
\end{tabular}


BCG: Calmette and Guérin bacillus; IQR: interquartile range; IR: isoniazid and rifampicin; IRE: isoniazid, rifampicin, and ethambutol; RIVM: Rijksinstituut voor Volksgezondheid en Milieuhygiene; SD: standard deviation. 